\title{
Front Matter: Volume 8599
}

, "Front Matter: Volume 8599," Proc. SPIE 8599, Solid State Lasers XXII: Technology and Devices, 859901 (3 April 2013); doi: 10.1117/12.2024877

SPIE. Event: SPIE LASE, 2013, San Francisco, California, United States 


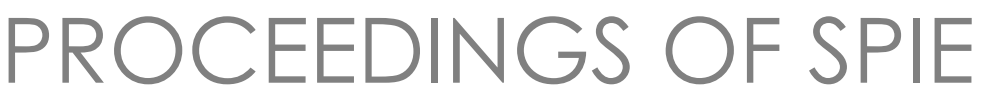

\title{
Solid State Lasers XXII: Technology and Devices
}

\author{
W. Andrew Clarkson \\ Ramesh K. Shori \\ Editors
}

3-5 February 2013

San Francisco, California, United States

Sponsored and Published by

SPIE 
The papers included in this volume were part of the technical conference cited on the cover and title page. Papers were selected and subject to review by the editors and conference program committee. Some conference presentations may not be available for publication. The papers published in these proceedings reflect the work and thoughts of the authors and are published herein as submitted. The publisher is not responsible for the validity of the information or for any outcomes resulting from reliance thereon.

Please use the following format to cite material from this book:

Author(s), "Title of Paper," in Solid State Lasers XXII: Technology and Devices, edited by

W. Andrew Clarkson, Ramesh K. Shori, Proceedings of SPIE Vol. 8599 (SPIE, Bellingham, WA, 2013) Article CID Number.

ISSN: 0277-786X

ISBN: 9780819493682

Published by

SPIE

P.O. Box 10, Bellingham, Washington 98227-0010 USA

Telephone +1 3606763290 (Pacific Time) · Fax +1 3606471445

SPIE.org

Copyright @ 2013, Society of Photo-Optical Instrumentation Engineers.

Copying of material in this book for internal or personal use, or for the internal or personal use of specific clients, beyond the fair use provisions granted by the U.S. Copyright Law is authorized by SPIE subject to payment of copying fees. The Transactional Reporting Service base fee for this volume is $\$ 18.00$ per article (or portion thereof), which should be paid directly to the Copyright Clearance Center (CCC), 222 Rosewood Drive, Danvers, MA 01923. Payment may also be made electronically through CCC Online at copyright.com. Other copying for republication, resale, advertising or promotion, or any form of systematic or multiple reproduction of any material in this book is prohibited except with permission in writing from the publisher. The CCC fee code is 0277-786X/13/\$18.00.

Printed in the United States of America.

Publication of record for individual papers is online in the SPIE Digital Library.

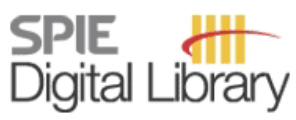

SPIEDigitalLibrary.org

Paper Numbering: Proceedings of SPIE follow an e-First publication model, with papers published first online and then in print and on CD-ROM. Papers are published as they are submitted and meet publication criteria. A unique, consistent, permanent citation identifier (CID) number is assigned to each article at the time of the first publication. Utilization of CIDs allows articles to be fully citable as soon as they are published online, and connects the same identifier to all online, print, and electronic versions of the publication. SPIE uses a six-digit CID article numbering system in which:

- The first four digits correspond to the SPIE volume number.

- The last two digits indicate publication order within the volume using a Base 36 numbering system employing both numerals and letters. These two-number sets start with 00, 01, 02, 03, 04, $05,06,07,08,09,0 A, 0 B \ldots 0 Z$, followed by 10-1Z, 20-2Z, etc.

The CID Number appears on each page of the manuscript. The complete citation is used on the first page, and an abbreviated version on subsequent pages. Numbers in the index correspond to the last two digits of the six-digit CID Number. 


\title{
Contents
}

\author{
xi Conference Committee
}

\section{SESSION 1 WAVEGUIDE LASERS I}

859902 Fluoride waveguide lasers grown by liquid phase epitaxy (Invited Paper) [8599-1]

F. Starecki, W. Bolaños, G. Brasse, A. Benayad, J.-L. Doualan, A. Braud, R. Moncorgé,

P. Camy, CIMAP, CNRS, Univ. de Caen (France)

$859904 \quad 2-\mu \mathrm{m}$ waveguide lasers in monoclinic double tungstates (Invited Paper) [8599-3]

J. J. Carvajal, C. J. Berrospe, X. Mateos, M. Aguilò, F. Díaz, Univ. Rovira i Virgili (Spain)

859905 Low-threshold, mirrorless emission at $981 \mathrm{~nm}$ in an Yb,Gd,Lu:KYW inverted rib waveguide laser [8599-4]

A. Choudhary, Univ. of Southampton (United Kingdom); W. Bolaños, Univ. Rovira i Virgili (Spain); P. Kannan, Univ. of Southampton (United Kingdom); J. J. Carvajal, M. Aguilò,

F. Diaz, Univ. Rovira i Virgili (Spain); D. P. Shepherd, Univ. of Southampton (United Kingdom)

\section{SESSION 2 WAVEGUIDE LASERS II}

859907 Versatile fs laser-written glass chip lasers (Invited Paper) [8599-6]

D. G. Lancaster, The Univ. of Adelaide (Australia); S. Gross, A. Fuerbach, Macquarie Univ. (Australia); H. Ebendorff Heidepriem, T. M. Monro, The Univ. of Adelaide (Australia);

M. J. Withford, Macquarie Univ. (Australia)

859908 Er-doped planar waveguides for power amplifier applications [8599-7]

J. I. Mackenzie, G. S. Murugan, The Univ. of Southampton (United Kingdom); A. W. YU,

J. B. Abshire, NASA Goddard Space Flight Ctr. (United States)

859909 Rare-earth-ion-doped ultra-narrow-linewidth lasers on a silicon chip and applications to intra-laser-cavity optical sensing [8599-8]

E. H. Bernhardi, R. M. de Ridder, K. Wörhoff, M. Pollnau, Univ. Twente (Netherlands)

SESSION 3 EYESAFE AND MID-IR LASERS I

8599 OB High-energy, $\mathrm{kHz}-\mathrm{rate}$, picosecond, 2- $\mu \mathrm{m}$ laser pump source for mid-IR nonlinear optical devices [8599-10]

A. Dergachev, Q-Peak, Inc. (United States)

8599 OC A broadly tunable continuous-wave Fe:ZnSe laser [8599-11]

J. W. Evans, P. A. Berry, K. L. Schepler, Air Force Research Lab. (United States)

8599 OD Gain-switched single-pass Cr:ZnSe amplifier [8599-12]

S. A. McDaniel, Scientific Applications International Corp. (United States); P. A. Berry,

K. L. Schepler, Air Force Research Lab. (United States) 
8599 OE Fe:ZnSe laser oscillation under cryogenic and room temperature [8599-13]

H. Jelínková, Czech Technical Univ. in Prague (Czech Republic); M. E. Doroshenko, A. M. Prokhorov General Physics Institute (Russian Federation); M. Jelínek, D. Vyhlídal, J. Šulc, M. Němec, V. Kubeček, Czech Technical Univ. in Prague (Czech Republic); Y. A. Zagoruiko, N. O. Kovalenko, A. S. Gerasimenko, V. M. Puzikov, V. K. Komar, The National Academy of Sciences of Ukraine (Ukraine)

8599 OG Efficient Er:YAG lasers at $1645.55 \mathrm{~nm}$, resonantly pumped with narrow bandwidth diode laser modules at $1532 \mathrm{~nm}$, for methane detection [8599-15]

H. Fritsche, O. Lux, C. Schuett, Technische Univ. Berlin (Germany); S. Heinemann, W. Gries, DirectPhotonics Industries GmbH (Germany); H. J. Eichler, Technische Univ. Berlin (Germany)

$8599 \mathrm{OH}$ Simulations and experiments on resonantly-pumped single-frequency Erbium lasers at 1.6 um [8599-16]

A. Meissner, P. Kucirek, Fraunhofer-Institut für Lasertechnik (Germany); J. Li, S. Yang, Beijing Institute of Technology (China); M. Hoefer, D. Hoffmann, Fraunhofer-Institut für Lasertechnik (Germany)

\section{SESSION 4 EYESAFE AND MID-IR LASERS II}

8599 Ol Coherent polarization locking of Q-switched Ho:YAG laser [8599-17]

C. F. Chua, L. H. Tan, P. B. Phua, Nanyang Technological Univ. (Singapore)

8599 0J Doped sesquioxide ceramic for eye-safe solid state laser materials [8599-18] W. Kim, C. Baker, U.S. Naval Research Lab. (United States); C. Florea, Sotera Defense Solutions (United States); J. Frantz, G. Villalobos, B. Shaw, S. Bowman, S. O'Connor, U.S. Naval Research Lab. (United States); B. Sadowski, Sotera Defense Solutions (United States); M. Hunt, Univ. Research Foundation (United States); I. Aggalwar, Sotera Defense Solutions (United States); J. Sanghera, U.S. Naval Research Lab. (United States)

8599 OK Supercontinuum generation in mid-IR using chalcogenide and germanate nonlinear fiber [8599-20]

N. Tolstik, Norwegian Univ. of Science and Technology (Norway); E. Sorokin, V. Kalashnikov, Technische Univ. Wien (Austria); D. Klimentov, V. Dvoyrin, I. T. Sorokina, Norwegian Univ. of Science and Technology (Norway)

$8599 \mathrm{OL}$ Design of a rugged $308 \mathrm{~nm}$ tunable UV laser for airborne LIF measurements on top of Zeppelin NT [8599-21]

M. Strotkamp, A. Munk, B. Jungbluth, Fraunhofer-Institut für Lasertechnik (Germany); K. Dahlhoff, P. Jansen, S. Broch, S. Gomm, M. Bachner, H. Fuchs, F. Holland,

A. Hofzumahaus, Forschungszentrum Jülich GmbH (Germany)

8599 ON Space-based, multi-wavelength solid-state lasers for NASA's Cloud Aerosol Transport System for International Space Station (CATS-ISS) [8599-23]

T. Chuang, P. Burns, E. B. Walters, T. Wysocki, T. Deely, A. Losse, K. Le, B. Drumheller,

T. Schum, M. Hart, K. Puffenburger, B. Ziegler, F. Hovis, Fibertek, Inc. (United States) 
$859900 \quad$ Space qualified laser transmitter for NASA's ICESat-2 mission [8599-24]

N. W. Sawruk, Fibertek, Inc. (United States); M. A. Stephen, NASA Goddard Space Flight Ctr. (United States); S. Litvinovitch, J. E. Edelman, M. M. Albert, R. E. Edwards, C. F. Culpepper,

W. J. Rudd, E. Fakhoury, F. E. Hovis, Fibertek, Inc. (United States)

8599 OP A 16-beam non-scanning swath mapping laser altimeter instrument [8599-25]

A. W. Yu, M. A. Krainak, D. J. Harding, J. B. Abshire, X. Sun, L. Ramos-Izquierdo,

J. Cavanaugh, S. Valett, T. Winkert, M. Plants, C. Kirchner, B. Kamamia, R. Faulkner,

P. Dogoda, W. Hasselbrack, T. Filemyr, NASA Goddard Space Flight Ctr. (United States)

\section{SESSION 6 PULSED LASERS}

$85990 Q \quad$ A highly energetic, multiwavelength, diode-pumped nanosecond laser system with flexible pulse-shaping capability [8599-26]

A. V. Okishev, I. A. Begishev, R. Cuffney, S. Papernov, J. D. Zuegel, Univ. of Rochester (United States)

8599 OS Self-Q-switched Cr:LiCAF laser near 800 nm [8599-28]

E. Beyatli, A. Sennaroglu, Koç Univ. (Turkey); U. Demirbas, Uluslararasi Antalya Univ. (Turkey)

8599 OT Multi-output Q-switched solid-state laser using an intra-cavity MEMS micromirror array [8599-29]

R. Baver, W. Lubeigt, Univ. of Strathclyde (United Kingdom); C. Clark, E. McBrearty, Helia Photonics Ltd. (United Kingdom); D. Uttamchandani, Univ. of Strathclyde (United Kingdom)

8599 OX Flashlamp failure modes and lifetime estimation techniques [8599-33]

R. J. F. Tucker, N. Cochran, G. L. Morelli, Honeywell Federal Manufacturing \& Technologies, LLC (United States)

\section{SESSION 7 LASER MATERIALS AND CHARACTERIZATION}

8599 OY Simulation of solid-state lasers with composites and ceramic crystals [8599-34] Z. Rahimi, Friedrich-Alexander-Univ. Erlangen-Nürnberg (Germany) and ASLD GmbH (Germany); C. Pflaum, Friedrich-Alexander-Univ. Erlangen-Nürnberg (Germany)

$85990 Z$ Thermo-optic quality assessment of doped optical ceramics [8599-35] C. C. C. Willis, J. D. Bradford, E. Maddox, L. Shah, M. Richardson, CREOL, The College of Optics and Photonics, The Univ. of Central Florida (United States)

859911 Optical and spectroscopic properties of Ytterbium-doped YAG [8599-37] N. D. Haynes, D. E. Zelmon, Air Force Research Lab. (United States)

859912 Quasi-single-mode random lasing within a ZnO nanoparticle film [8599-38] H. Fujiwara, R. Niyuki, Hokkaido Univ. (Japan); Y. Ishikawa, Kagawa Univ. (Japan); N. Koshizaki, National Institute of Advanced Industrial Science and Technology (Japan); T. Tsuji, Kyushu Univ. (Japan); K. Sasaki, Hokkaido Univ. (Japan) 
859914 Energy scaling of a multipass-cavity mode-locked femtosecond bulk laser with a carbon nanotube saturable absorber [8599-41]

I. Baylam, Koç Univ. (Turkey); S. Ozharar, Bahçesehir Univ. (Turkey); H. Cankaya, Koç Univ. (Turkey); S. Y. Choi, K. Kim, F. Rotermund, Ajou Univ. (Korea, Republic of); U. Griebner, V. Petrov, Max-Born-Institut für Nichtlineare Optik und Kurzzeitspektroskopie (Germany); A. Sennaroglu, Koç Univ. (Turkey)

$859915 \quad 200 \mathrm{~W}$ fs Innoslab amplifier with 400 $\mu \mathrm{J}$ pulse energy for industrial applications [8599-42] T. Mans, AMPHOS GmbH (Germany); C. Hönninger, Amplitude Systèmes (France); J. Dolkemeyer, AMPHOS GmbH (Germany); A. Letan, Amplitude Systèmes (France); C. Schnitzler, AMPHOS GmbH (Germany); E. Mottay, Amplitude Systèmes (France)

8599161 Watt femtosecond mid-IR Cr:ZnS laser [8599-43]

E. Sorokin, Technische Univ. Wien (Austria); N. Tolstik, I. T. Sorokina, Norwegian Univ. of Science and Technology (Norway)

859919 Repetitive petawatt-class laser with near-diffraction-limited focal spot and transformlimited pulse duration [8599-46]

C. Liu, S. Banerjee, J. Zhang, S. Chen, K. Brown, J. Mills, N. Powers, B. Zhao, G. Golovin, I. Ghebregziabher, D. Umstadter, Univ. of Nebraska-Lincoln (United States)

\section{SESSION 9 POWER SCALING BULK SSL AND FIBER LASERS: JOINT SESSION WITH CONFERENCES 8599 AND} 8601

8599 1B Multi-kW high brightness Yb:YAG thin disk laser [8599-48]

Y. H. Peng, Y. X. Lim, J. Cheng, Y. B. Tan, E. Lau, K. S. Lai, DSO National Labs. (Singapore)

8599 1C 200-W Tm:YLF INNOSLAB laser [8599-49]

A. Meissner, Fraunhofer-Institut für Lasertechnik (Germany); J. Li, Beijing Institute of Technology (China); I. Lopez- Perez, Fraunhofer-Institut für Lasertechnik (Germany);

S. Yang, Beijing Institute of Technology (China); M. Hoefer, D. Hoffmann, Fraunhofer-Institut für Lasertechnik (Germany)

\section{SESSION 10 NOVEL CONCEPTS}

8599 1D Wavelength dependence of the optical axis in double tungstate crystals [8599-50] R. Cattoor, Institut Franco-Allemand de Recherches de Saint-Louis (France) and Lab. Ondes et Matière d'Aquitaine (France); I. Manek-Hönninger, M. Tondusson, Lab. Ondes et Matière d'Aquitaine (France); T. Kalkandjiev, Conerefringent Optics SL (Spain); D. Rytz, FEE GmbH (Germany); L. Canioni, Lab. Ondes et Matière d'Aquitaine (France); M. Eichhorn, Institut Franco-Allemand de Recherches de Saint-Louis (France)

$85991 \mathrm{G}$ Resonant optical devices for IR lasers [8599-53]

E. G. Johnson, Y. Li, I. Raghu Srimathi, R. H. Woodward, M. K. Poutous, A. J. Pung, Clemson Univ. (United States); M. Richardson, L. Shaw, CREOL, The College of Optics and Photonics, Univ. of Central Florida (United States); R. Shori, Naval Air Warfare Ctr. Weapons Div. (United States); R. Magnusson, The Univ. of Texas at Arlington (United States) 
$8599 \mathrm{lH} \quad 879 \mathrm{~nm}$ pump diode stack and single Nd:YAG rod design to achieve $20 \mathrm{~W}$ to $300 \mathrm{~W}$ adjustable laser output power at $532 \mathrm{~nm}$ and $38 \%$ optical to optical conversion efficiency [8599-54]

J. R. Xuan, M. Scott, X. Yang, T. C. Hasenberg, American Medical Systems (United States)

\section{SESSION $11 \quad$ UV-VIS LASERS}

8599 11 High power high repetition rate VCSEL array side-pumped pulsed blue laser [8599-55]

R. van Leeuwen, P. Zhao, T. Chen, B. XU, L. Watkins, J.-F. Seurin, G. XU, A. Miglo, Q. Wang,

C. Ghosh, Princeton Optronics, Inc. (United States)

$85991 \mathrm{~J} \quad$ 0.9W compact UV pulsed lasers using high-power VCSEL array side-pumping [8599-56] T. Chen, B. Xu, R. Van Leeuwen, P. Zhao, J.-F. Seurin, G. Xu, A. Miglo, Q. Wang, C. Ghosh, Princeton Optronics, Inc. (United States)

8599 1K Laser and phosphor hybrid source for projection display [8599-57]

F. Hu, Y. Li, Appotronics Corp. (China)

8599 IL Wide temperature operation of a VCSEL pumped 355nm frequency tripled Nd:YAG laser [8599-58]

B. Cole, A. Hays, C. Mclntosh, L. Goldberg, U.S. Army RDECOM Communications-

Electronics, Development and Engineering Ctr. (United States)

\section{SESSION 12 DISK LASERS}

8599 1M Cr:ZnSe thin disk cw laser [8599-59]

G. Renz, J. Speiser, A. Giesen, Deutsches Zentrum für Luft- und Raumfahrt e.V. (Germany); I. T. Sorokina, Norwegian Univ. of Science and Technology (Norway); E. Sorokin, Technische Univ. Wien (Austria)

8599 iN High-power Kerr-lens mode-locked thin-disk oscillator in the anomalous and normal dispersion regimes [8599-60]

O. Pronin, Ludwig-Maximilians-Univ. München (Germany); J. Brons, M. Seidel, Max-PlanckInstitut für Quantenoptik (Germany); F. Lücking, Ludwig-Maximilians-Univ. München (Germany); C. Grasse, G. Boehm, M. C. Amann, Walter Schottky Institut (Germany); V. Pervak, Max-Planck-Institut für Quantenoptik (Germany); A. Apolonski, LudwigMaximilians-Univ. München (Germany) and Max-Planck-Institut für Quantenoptik (Germany); V. L. Kalashnikov, Technische Univ. Wien (Austria); F. Krausz, LudwigMaximilians-Univ. München (Germany) and Max-Planck-Institut für Quantenoptik (Germany)

859910 Erbium-based edge-pumped disk laser [8599-61]

J. Vetrovec, D. A. Copeland, A. S. Litt, Aqwest, LLC (United States); D. Du, General Atomics Aeronautical Systems, Inc. (United States)

8599 IP Amplified spontaneous emission (ASE) models and approximations for thin-disk laser modeling [8599-62]

D. A. Copeland, Aqwest, LLC (United States) 
8599 1Q Zero-phonon-line pumped 1 kHz Yb:YAG thin-disk regenerative amplifier [8599-63] M. Chyla, Institute of Physics of the ASCR, v.v.i. (Czech Republic) and Czech Technical Univ. in Prague (Czech Republic); T. Miura, M. Smrz, Institute of Physics of the ASCR, v.v.i. (Czech Republic); P. Severova, O. Novak, Institute of Physics of the ASCR, v.v.i. (Czech Republic) and Czech Technical Univ. in Prague (Czech Republic); A. Endo, T. Mocek, Institute of Physics of the ASCR, v.v.i. (Czech Republic)

POSTER SESSION

8599 IR Solid-state laser source of narrowband ultraviolet B light for skin disease care [8599-64] A. A. Tarasov, H. Chu, Laseroptek (Korea, Republic of)

8599 is Power-scaling of $\mathrm{Pr}: \mathrm{YAlO}_{3}$ laser operating in $\mathrm{CW}$ regime at $747 \mathrm{~nm}$ and $720 \mathrm{~nm}$ wavelengths [8599-65]

M. Fibrich, Czech Technical Univ. in Prague (Czech Republic) and Institute of Physics (Czech Republic); H. Jelínková, Czech Technical Univ. in Prague (Czech Republic)

8599 IU Tunability of Yb:glass laser [8599-67]

J. Šulc, O. Krivosudský, H. Jelínková, Czech Technical Univ. in Prague (Czech Republic);

R. Stepien, Institute of Electronic Materials Technology (Poland)

8599 IV Cavity length dependence of mode beating in passively Q-switched Nd-solid state lasers [8599-68]

N. D. Zameroski, M. Wanke, D. Bossert, Sandia National Labs. (United States)

8599 IW High energy intracavity pumped eye-safe $\mathrm{BaWO}_{4}$ Raman laser [8599-69]

O. Kitzler, H. Jelínková, J. Šulc, L. Koubíková, M. Němec, Czech Technical Univ. in Prague

(Czech Republic); K. Nejezchleb, V. Škoda, Crytur Ltd. (Czech Republic)

8599 1X A low jitter single frequency Q-switched laser from solid state to optical fiber configuration [8599-71]

F. F. Wu, Consultant (United States)

859912 Observation of laser beam profile progression inside an extended laser cavity [8599-73]

F. F. Wu, MetroLaser Inc. (United States); T. C. Farrell, Air Force Research Lab. (United States)

859920 Exploring optical properties of $\mathrm{Nd}$-doped vanadates with intracavity self-mode locking [8599-74]

H. C. Liang, J. C. Tung, C. H. Tsou, K. W. Su, Y. F. Chen, National Chiao Tung Univ. (Taiwan)

$859921 \quad$ Cr:ZnMgSe laser pumped by $1.7 \mu \mathrm{m}$ Er:YLF radiation [8599-75]

M. E. Doroshenko, A. M. Prokhorov General Physics Institute (Russian Federation);

H. Jelínková, M. Němec, J. Šulc, M. Jelínek, Czech Technical Univ. in Prague (Czech

Republic); V. K. Komar, A. S. Gerasimenko, Institute for Single Crystals (Ukraine);

V. M. Puzikov, V. V. Badikov, Kuban State Univ. (Russian Federation)

859923 Characterization of polarizing splitter optics in extreme environments [8599-77]

R. J. F. Tucker, M. Olson, G. L. Morelli, Honeywell Federal Manufacturing \& Technologies,

LLC (United States) 
859926 High energy diode pumped 5th harmonic generation of Nd: YAG laser [8599-80]

Y. Yu, C. Y. Cheng, Y. P. Chia, W. H. Wong, S. S. Yong, W. Qu, Ngee Ann Polytechnic

(Singapore); X. Peng, Enlight Technologies, Inc. (United States)

$859927 \quad$ High efficient, high peak power of $18 \mathrm{~kW}$ with $4 \mathrm{~ns}$ pulses of diode pumped passive Qswitched and self $Q$-switched Nd: $\mathrm{YVO}_{4}$ laser at both $1064 \mathrm{~nm}$ using $\mathrm{Cr}: Y A G$ and $532 \mathrm{~nm}$ using KTP crystals [8599-81]

A. F. El-Sherif, CREOL, The College of Optics and Photonics, Univ. of Central Florida (United States) and Military Technical College (Egypt); M. M. Talaat, Military Technical Institute (Egypt)

859928 Development of kW class Nd:YAG composite ceramic thin disc laser [8599-82]

K. Iyama, Osaka Univ. (Japan), ALPROT (Japan) and Hamamatsu Photonics K.K. (Japan); R. Bhushan, Osaka Univ. (Japan); H. Furukawa, ALPROT (Japan) and ILT (Japan);

K. Tsubakimoto, H. Yoshida, H. Fujita, Osaka Univ. (Japan); M. Fujita, ALPROT (Japan) and ILT (Japan); N. Miyanaga, Osaka Univ. (Japan); Y. Tamaoki, Y. Kato, T. Kawashima, ALPROT (Japan) and Hamamatsu Photonics K.K. (Japan)

859929 Spectroscopic characterization and upconversion processes under $\sim 1.5 \mu \mathrm{m}$ pumping in Er doped Yttria ceramics [8599-39]

E. E. Brown, U. Hommerich, Hampton Univ. (United States); A. Bluiett, Elizabeth City State Univ. (United States); C. Kucera, J. Ballato, Clemson Univ. (United States); S. Trivedi, Brimrose Corp. of America (United States)

Author Index 
Proc. of SPIE Vol. $8599859901-10$

Downloaded From: https://www.spiedigitallibrary.org/conference-proceedings-of-spie on 26 Apr 2023 Terms of Use: https://www.spiedigitallibrary.org/terms-of-use 


\title{
Conference Committee
}

\author{
Symposium Chairs
}

Bo Gu, Bos Photonics (United States)

Andreas Tünnermann, Fraunhofer-Institut für Angewandte Optik und Feinmechanik (Germany) and Friedrich-Schiller-Universität Jena (Germany)

Symposium Cochairs

Friedhelm Dorsch, TRUMPF Werkzeugmaschinen GmbH + Co. KG (Germany)

Alberto Piqué, U.S. Naval Research Laboratory (United States)

Program Track Chair

Gregory J. Quarles, Optoelectronics Management Network (United States)

Conference Chairs

W. Andrew Clarkson, University of Southampton (United Kingdom) Ramesh K. Shori, Naval Air Warfare Center Weapons Division (United States)

\section{Conference Program Committee}

Patrick A. Berry, Air Force Research Laboratory (United States)

Marc Eichhorn, Institut Franco-Allemand de Recherches de Saint-Louis (France)

Dennis G. Harris, MIT Lincoln Laboratory (United States)

Norman Hodgson, Coherent, Inc. (United States)

Helena Jelínková, Czech Technical University in Prague (Czech Republic) Christian Kränkel, Universität Hamburg (Germany) Jacob I. Mackenzie, University of Southampton (United Kingdom) Markus Pollnau, Universiteit Twente (Netherlands)

Narasimha S. Prasad, NASA Langley Research Center (United States) Martin Richardson, CREOL, The College of Optics and Photonics, University of Central Florida (United States)

Akira Shirakawa, The University of Electro-Communications (Japan)

David H. Titterton, Defence Science and Technology Laboratory (United Kingdom) 


\section{Session Chairs}

1 Waveguide Lasers I

Dennis G. Harris, MIT Lincoln Laboratory (United States)

2 Waveguide Lasers II

Dennis G. Harris, MIT Lincoln Laboratory (United States)

3 Eyesafe and Mid-IR Lasers I

Marc Eichhorn, Institut Franco-Allemand de Recherches de Saint-Louis (France)

4 Eyesafe and Mid-IR Lasers II

Marc Eichhorn, Institut Franco-Allemand de Recherches de Saint-Lovis (France)

5 Airborne and Space Qualified Lasers

Narasimha S. Prasad, NASA Langley Research Center (United States)

6 Pulsed Lasers

Christian Kränkel, Universität Hamburg (Germany)

7 Laser Materials and Characterization

Jacob I. Mackenzie, University of Southampton (United Kingdom)

8 Ultrafast Lasers

Martin Richardson, CREOL, The College of Optics and Photonics, University of Central Florida (United States)

9 Power Scaling Bulk SSL and Fiber Lasers: Joint Session with Conferences 8599 and 8601

Eric C. Honea, Lockheed Martin Aculight (United States)

W. Andrew Clarkson, University of Southampton (United Kingdom)

10 Novel Concepts

Helena Jelínková, Czech Technical University in Prague (Czech Republic)

11 UV-VIS Lasers

Helena Jelínková, Czech Technical University in Prague (Czech Republic)

12 Disk Lasers

David H. Titterton, Defence Science and Technology Laboratory (United Kingdom) 